Elsworth, J. D., Dewar, D. Glover, V., Goodwin, B. L. Clow, A. \& SANDLER, M. (1986) Purification and characterisation of tribulin, an endogenous inhibitor of monoamine oxidase and of benzodiazepine receptor binding. Journal of Neural Transmission, 67, 45-56.

Petursson, H., Bhattacharya, S. K., Glover, V., Sandler, M and LADER, M. H. (1982) Urinary monoamine oxidase inhibitor and benzodiazepine withdrawal. British Journal of Psychiatry. 140, 7-10.

SANDLER, M. (1982) The emergence of tribulin. Trends in Pharmaco logical Sciences, 3, 471-472.

\section{HLA-DR2 and Sleep Onset REM Periods in} Endogenous Depression

SIR: With rare exceptions (Zietz et al, 1986), almost all patients with narcolepsy are HLA-DR2 positive (Billiard \& Seignalet, 1985), while the normal population frequency is about $20-35 \%$. To date, this association constitutes the strongest relationship between a given disease and the HLA-system.

Besides clinically relevant symptoms such as cataplexy, excessive daytime somnolence, hypnagogic hallucinations, and sleep paralysis, one main feature of narcolepsy is the occurrence of sleep onset REM periods (SOREMPs) (REM latency $\leq 25 \mathrm{~min}$ ) during daytime and night-time sleep. Recently, a correlation between length of REM latency and possession of HLA-DR2 was reported (Schulz et al, 1986). Healthy subjects who were HLA-DR2 positive exhibited significantly shorter REM latencies than those who were not positive. However, this result could not be replicated (Schulz et al, 1987).

The fact that narcoleptic patients and patients with endogenous depression share one common feature in their sleep pattern, i.e. the occurence of sleep onset REM periods, stimulated us to investigate HLA-DR2 in patients with endogenous depression, to clarify if there is a common genetic basis for this deviant sleep pattern.

We have investigated 11 patients with a major depressive disorder of the endogenous subtype according to Research Diagnostic Criteria (Spitzer et al, 1977). Seven of them were female, four were male. Mean age ( \pm s.d.) was $49.6 \pm 8.4$ years. Two of the patients were bipolar II, one patient was bipolar I. Blood samples for HLA-DR2 typing were drawn during a hospital stay and analysed using the microlymphocytotoxicity test according to the method of Terasaki.

All of the patients took part in ongoing sleep studies in our sleep laboratory, and had between two and five nights of baseline sleep recordings. The patients were free of any kind of psychoactive medication prior to sleep recording for at least seven days.

Seven of the patients had at least one sleep onset REM period. Four of them were HLA-DR2 positive, three were negative. Of the four patients showing no sleep onset REM periods, three were HLA-DR2 positive. Calculating a Fisher exact test for the occurrence of SOREMPs (yes/no) and HLA-DR2 positive/ negative, a $P$ value of 0.42 was attained. This result clearly contradicts the asumption that there is a close relationship between HLA-DR2 positive and sleep onset REM periods in endogenous depression.

Strikingly, however, the HLA-DR2 positive rate of $64 \%$ in our sample ( 7 of 11 patients were positive) of depressed patients by far exceeded the normal population rate of $20-35 \%$. Before definite conclusions can be drawn from this result, larger samples of depressed patients and patients with other psychiatric disorders must be investigated, to clarify whether this result is due to chance or sample selection.

Dieter RiemanN MATHIAS BERGER

Psychiatric Clinic

Central Institute of Mental Health

Mannheim

West Germany

2nd Medical Clinic

Mannheim

University of Heidelberg

\section{References}

Billiard, M. \& Seignalet, J. (1985) Extraordinary association between HLA-DR2 and narcolepsy. The Lancet, i, 226-227.

Schulz, H., Geisler, P., Pollmaecher, T., Zietz, A., Keller, E., SCHOLZ, S. \& ALBERT, E. (1986) HLA-DR2 correlates with rapid eye movement sleep latency in normal human subjects. The Lancet, ii, 803.

- (1987) HLA-DR2 and rapid eye movement sleep latency: failure to replicate. The Lancet, ii, 627.

Spitzer, R. L., Endicotr, J. E. \& Robins, E. (1977) Research Diagnostic Criteria for a Selected Group of Functional Disorders (3rd edn). New York: National Psychiatric Institute, Biometric Research Department.

Zietz, A., Keller, E., Scholz, S., Albert, E., Roth, B., Nevimalowa, S., Sonka, K., Docekal, P., Ivaskova, E., SCHULz, H. \& GeISLER, P. (1986) DR2-negative narcolepsy. The Lancet, ii, 684-685.

\section{Mystical-Ecstatic and Trance States}

SIR: Mystical-ecstatic and trance states fall within the general category of depersonalisation symptom and dissociative states. Usually they are pleasant, relaxing, short-lived, and do not have psychopathological consequences (Benson, 1984).

Case report: $\mathrm{Mr}$ A, a 26-year-old right-handed man, had a life-long interest in mystical and religious philosophy, 
eventually joining a Cistercian monastery for two years. In 1981 he had become frightened by several intense meditative experiences, giving up the practice for some time. In September 1986 he entered a prolonged ecstatic state with heightened awareness, and increased attention and concentration that he believed was necessary to develop his psychic powers. It was initially pleasant and thought to be derived from spirituality and the contemplation of God.

However, as this state became a constant feature, Mr A found he could no longer control it or make it go away and he became mildly depressed with fatigue, $5 \mathrm{lb}$ weight loss, and awakening from vivid dreams. During this time he had five panic attacks, lasting only a few minutes but preceded by 1-3 days of anxiety. He had one short previous episode of situational depression of moderate severity. His father is a chronic alcoholic, and both a brother and sister have been treated for depression. On presentation, Mr A's mood was mildly anxious and affect slightly restricted; there was no evidence of psychosis.

He showed mild left frontal lobe signs with decreased word list generation and perseveration with a reciprocal hand sequence test; deep tendon reflexes were increased on the right, and he showed dystonic posturing on that side with a complex gait task. The Wechsler Adult Intelligence Scale showed an above-average score (Verbal 118, Performance 105); personality testing gave evidence of general distress and interpersonal isolation.

$\mathrm{Mr}$ A was initially unsure whether he was willing to give up the mystical ecstasy in exchange for relief of the panics; hyperventilation on occasion had brought on a state that resembled meditation. Alprazolam helped somewhat, but increased the euphoric dissociation causing a sensation of levitation and "out of body" experiences; when phenelzine was substituted, the ecstatic state, panics, and mood change lifted in three weeks.

The predominant feature of his complaint was the feeling of strangeness or change which was initially pleasant and desired, and long preceded those akin to 'atypical' depression, or the few panic attacks. The picture resembles the syndrome of intellectualobsessive depersonalisation with endless ruminative self-scrutiny (Torch, 1978). Depersonalisation disorder has probably the weakest description in the current DSM-III nosology, even though the symptom occurs frequently in dissociative, affective, and anxiety disorders as well as in psychosis. Attempts to define the syndrome essentially ended after the delineation of phobic anxiety-depersonalisation syndrome, although there was a slight revival with the study of meditation and 'transcendental' states. The salient features are: a subjective awareness of a feeling of change, strangeness, or unreality; a quality of unpleasantness or distress; loss of affective responsiveness; and the nondelusional quality (Ackner, 1954). Agents with sedating effects usually worsen depersonalisation; an MAOI was selected because of the class's stimulating effects and proven benefit with mixed anxiety-depressive states, panic disorders, and atypical depression.

The phenomenology, associated conditions, and treatment of depersonalisation disorder must be better described if it is to survive as a useful diagnostic entity.

\section{Royal Ottowa Hospital \\ Ontario K1Z $7 K 4$}

Canada

\section{References}

ACKNER, B. (1954) Depersonalization: I. Aetiology and phenomenology. Journal of Mental Science, 100, 838-853.

Benson, H. (1984) The relaxation response and the treatment of anxiety. In Psychiatry Update Vol. III (ed. L. Grinspoon). Washington, DC: American Psychiatric Press.

TORCH, E. M. (1978) Review of the relationship between obsession and depersonalization. Acta Psychiatrica Scandinavica, 58, 191-198.

\section{Unilateral Auditory Hallucinations}

SIR: There have been a few reports of unilateral complex auditory hallucinations, and this lateralisation phenomenon has been attributed to the brain lesion contralateral to the side of the hallucinations. We report a patient with unilateral auditory hallucinations related to deafness on the same side.

Case report: A 72-year-old lady presented with a 26-year history of auditory hallucinations which had become louder and distressing over the preceding two months. The hallucinations were of complex verbal form. They were also stereotyped and repetitive in character. The voices were located in objective space and were attributed to her mother. The hallucinations were restricted to the left ear. There was associated depression, but this was in response to the persistence of the hallucinations. No other psychopathology was evident on mental state examination, and her personality was intact. She was originally seen in 1964, and was diagnosed as suffering from schizophrenia. She had consequently been treated with neuroleptics ever since. However, to our knowledge there had been no evidence of psychopathology pathognomic of schizophrenia. She had a childhood history of otis media with resultant deafness in the left ear since the age of seven. Her physical examination and routine blood investigations were unremarkable except for evidence of tardive dyskinesia. Recording of her EEG was normal. Hearing tests revealed that she had conductive deafness of the left ear.

On this admission the working hypothesis was that her unilateral auditory hallucinations resulted from her deafness. She was therefore encouraged to use her hearing aid. Indeed, she had been provided with a hearing aid for a number of years and had rarely used it, because she felt it was socially embarassing. As her compliance improved, her auditory hallucinations disappeared. She became cheerful and fit enough to discharge. She had no medication. 